\title{
ERRATUM: Scaling Behavior of Surface Irregularity in the Molecular Domain: From Adsorption Studies to Fractal Catalysts
}

\author{
J. Stat. Phys. 36:699 (1984).
}

\section{Peter Pfeifer, ${ }^{1}$ David Avnir, ${ }^{2}$ and Dina Farin ${ }^{2}$}

1. The measurements shown in Fig. 2 refer to adsorption of ethanol, not tertiary amyl alcohol. Accordingly, sentences No. 2 and 4 in the second paragraph of Section 3 should be changed to: "The reference yardstick chosen was ethanol... By Eqs. (4) and (5), this implies (1), with $D=$ $3.04 \pm 0.05$, over the yardstick range of $23-168 \AA^{2}$."

2. Contrary to what appears to be implied by Steinhaus in his paper and has been taken for granted in ours, the Steinhaus length of a curve, Eq. (9), is not unconditionally equal to the curve length measured with yardsticks of length $r$. This follows from numerical studies by M. Obert and $\mathrm{H}$. Wippermann (personal communication) for a highly branched dendritic curve. Also, the theorem that in the plane a straight line intersects a $D$-dimensional curve in a set of dimension $D-1$ with probability one $(\mathrm{P}$. Mattila, Ann. Acad. Sci. Fenn. Ser. AI 1:227 (1975); and references therein) implies that in Eq. (9) typically $S_{1}=\cdots=S_{m}=\infty$ for a fully developed fractal curve (zero inner cutoff). So, the general relationship between Steinhaus length as function of the spacing $r$, and length measured with yardsticks of length $r$, is to be clarified yet. Accordingly it may turn out that the domain of validity of Eq. (10) is restricted to $D \rightarrow 2$ and nonzero inner cutoff.

\footnotetext{
${ }^{1}$ Fakultät für Chemie, Universität Bielefeld, D-4800 Bielefeld, West Germany.

${ }^{2}$ Department of Organic Chemistry, The Hebrew University of Jerusalem, Jerusalem 91904, Israel.
} 